\title{
Short Report \\ Exploring Ethnicity in Hospital Patients with COVID-19 in South London
}

\begin{abstract}
Ethnicity was found to be an independent risk factor in COVID-19 outcomes in the UK and USA during the pandemic surge. London, being in the epicentre and having one of the most ethnically diverse population in the UK, was likely to have experienced a much higher intensity of this phenomenon. Black Asian and Minority ethnic groups were more likely to be admitted, more likely to require admission to intensive care and more likely to die from COVID-19. We undertook an analysis of a case series to explore the impact of ethnicity in hospitalised patients with confirmed COVID-19 during the 3 months of the pandemic. Our results demonstrated that although the proportion of Asian and Black patients were representative of the local population distribution, they were much younger. The prevalence of comorbidities was similar but logistic regression analysis showed that male sex (OR 1.4, 95\% $\mathrm{Cl} 1.1-1.9 ; \mathrm{p}=0.02$ ), age (OR 1.03, 95\% Cl 1.02 - 1.04, $\mathrm{p}<0.001$ ), those in the 'Other' [Odds ratio 1.7 (1.12.6) $\mathrm{p}=0.01$ ] and 'Asian'[Odds ratio $1.8(1.1-2.7) \mathrm{p}=0.01$ ], category were at higher risk of death in this cohort. Our results, therefore, are consistent with the overall data from the UK and USA indicating that ethnicity remains a significant additional risk and hence our clinical services must ensure that adequate provision is made to cater for this risk and research must be designed to understand the causes.
\end{abstract}

Key words

COVID-19, mortality, ethnicity, health inequalities
Anna Zatorska BMBS, Niladri Konar MRCP1, Pratyasha Saha MB BChir, Alice Moseley MBBS, Jessica Denman MSc, Reema Ali MSc, Fabiola Hatahintwali MSc and Indranil Chakravorty PhD

St Georges University Hospital NHS Trust, London, UK

1 Royal Alexandra Hospital, Harlow, UK

Correspondence to

Anna.zatorska@stgeorges.nhs.uk

Cite as: Zatorska, A., Konar, N., Saha, P., Moseley, A., Denman, J., Ali, R., Hatahintwali, F., Chakravorty, I. Exploring ethnicity in hospital patients with COVID-19. The Physician vol6; issue 2; epub 17.08.2020 DOI: https://doi.org/10.38192/1.6.2.16

Article Information

Submitted $\quad 16.08 .2020$

Pre-print $\quad 17.08 .2020$

Open Access - Creative Commons Licence CCBY-ND-4.0

\section{Open Access}

\section{Background}

At the beginning of the COVID-19 pandemic in China, predictors of worse disease related outcomes were male sex, advanced age and cardiovascular comorbidities. However, the global progression of the SARS-CoV-2 virus resulted in emerging data on the impact of ethnicity across the United Kingdom (UK) and United States of America (1). During the surge in UK cases, local centres saw a high incidence of intensive care unit (ITU) admissions and mortality rates in Black, Asian and Minority (BAME) ethnic backgrounds; this identified a need for further research (1)(2). A comprehensive analaysis of National Health Service (NHS) Digital databases which included approximately 80,000 patients, showed that individuals from a BAME background were more likely to be diagnosed with COVID-19, more likely to be admitted to hospital and intensive care, and more likely to die in comparison to general population (3). BAME background emerged as an independent risk factor for the pandemic, in particularly South Asian ethnicity (2)(4). Although BAME patients affected by COVID-19 were younger and with significantly lower median ages (Asian 51, Mixed/Other 57, Black 57 years old) than white patients ( 69 years old), the total burden of comorbidities was similar across all ethnicities. Additionally, respiratory diseases were more prevalent in White patients whilst cardiovascular and endocrine diseases were more prevalent in BAME patients. Increased prevalence of COVID-19 amongst individuals from a BAME background may be explained by the geographical distribution of the virus in the UK, in 
addition to deprivation and occupational exposure. The UK is a country with a large ethnically diverse population, and analysis can therefore contribute to our understanding of the disease's effects in various ethnic groups; the ethnic minority population of the UK was around $13 \%$ at the time of the last census in 2011(5). Greater London and the South East have the highest number of people who were born outside of the UK, out of which almost a third speak a language other than English as their first language. Furthermore, London's ethnically diverse population includes 18.5\% Asian, 13.3\% Black, 5\% Mixed, 3.4\% Other and $44.9 \%$ White ethnic groups(6). Thus, the impact of ethnicity on COVID-19 outcomes in Greater London is likely to be more apparent than the UK as a whole. Being at the epicentre of the pandemic in the UK, our Hospital experienced the early surge of patients affected by COVID19 with a total number of 855 cases between March and May. This report aims to explore the impact of ethnicity on COVID-19 outcomes in order to improve current clinical practice.

\section{Aim}

To explore the relationship between ethnicity and health related outcomes in patients with COVID-19.

\section{Design \& Methods}

A retrospective review of patient case notes of those with a polymerase chain reaction (PCR) confirmed SARS-CoV-2 infection, diagnosed March-May 2020 in St George's Hospital, London. The data collection was registered as an institutional audit (registration number: 10051). Patients were included if they were admitted overnight. Exclusion criteria were age below 16 years old and being employed by our Trust. Data collection included: demographics, ethnicity, length of stay, history of co-morbidities. The primary outcome was death or discharge.

Ethnicity was defined as per UK census definition and from NHS records as $0=$ not known, $1=$ Asian, $2=$ Black, $3=$ Mixed, $4=$ Other ethnicity and $5=$ White. Age on admission was classified into categories; $1=<40$ years, $2=40-49,3=$ $50-59,4=60-69$ and $5=>70$ years. The data were collected from hospital electronic records, anonymised and analysed using SPSS statistical software (SPSS v26, IBM Inc, USA). Data analysis included descriptive statistics and binary logistic regression modelling.

\section{Results}

Data collection identified confirmed 1966 SARS-CoV-2 cases between March and May 2020. Inclusion and exclusion criteria were applied, narrowing this to 1016 cases. 161 patients were admitted more than once, thus the final data analysis included 855 patients.

This cohort consisted of 470 (55\%) men and 385 (45\%) women. 419 (49\%) patients were from Asian, Black, Mixed and Other backgrounds, whilst 513 (59.8\%) were white. For 141 (16.4\%) patients ethnicity was unknown (table 1). The mean age of male patients in our sample was 67.1 ( $S D=16.4$ ) years whilst the mean age of women was $67.7(S D=18.8)$ years. Figure 1 shows the distribution of age in each of the ethnic groups with Asian (mean 60.8, SD 17.8 years for men and mean 61.9, SD 19.8 years for women) and Black women (mean 63.8, SD 19.1 years) being younger in this cohort compared to White ethnic group (mean 70.1, SD 15.5 years for men and mean 71.2, SD 18.2 years for women). In total, 295 patients died whilst 560 survived to discharge (figure 3). The length of stay in hospital was 12.2 ( $S D=11.3$ ) days for men and $11.9(S D=10.9$ ) days for women (table1). Figure 2 shows the proportion of patients in each age group with comorbidities. There was a rising trend for the presence of comorbidities with increasing age band. Binary logistic regression analysis showed that male sex (OR $1.4,95 \% \mathrm{Cl}$ 1.1-1.9; $p=0.02$ ), age (OR 1.03, 95\% Cl $1.02-1.04$, $p<0.001)$, those in the 'Other' [Odds ratio $1.7(1.1-2.6) p=$ 0.01 ] and 'Asian' [Odds ratio 1.8 (1.1-2.7) $p=0.01$ ], category were independent predictors of death in this cohort.

Table 1 shows the proportions in each ethnic group for gender, comorbidities and death/

\begin{tabular}{|l|l|l|l|l|l|l|l|}
\hline Category & $\begin{array}{l}\text { Ethnic- not } \\
\text { known (\%) }\end{array}$ & Ethnic -Asian & $\begin{array}{l}\text { Ethnic - } \\
\text { Black }\end{array}$ & $\begin{array}{l}\text { Ethnic } \\
\text { Mixed }\end{array}$ & $\begin{array}{l}\text { Ethnic } \\
\text { Other }\end{array}$ & $\begin{array}{l}\text { Ethnic- } \\
\text { White }\end{array}$ \\
\hline London population & na & 18.5 & 13.3 & 5 & 3.4 & 59.8 \\
\hline
\end{tabular}


International Journal of Health

\begin{tabular}{|l|l|l|l|l|l|l|}
\hline Proportion (\%) & 16.4 & 16.4 & 14.1 & 2.2 & 16.4 \\
\hline Men (\%) & 60 & 60.5 & 48.2 & 50 & 56.3 \\
\hline Age (SD) years & $66.7(16.7)$ & $61.2(18.5)$ & $67.1(17.9)$ & $62(18.7)$ & $68.5(16.6)$ & $70.7(16.8)$ \\
\hline LoS (SD) days & $13.2(11.9)$ & $10.6(10.9)$ & $12.9(12.3)$ & $8.6(7.6)$ & $12.9(11.3)$ & $11.8(10.4)$ \\
\hline Comorbidities (\%) & 51.8 & 50 & 68.6 & 73.3 & 61.6 & 53.4 \\
\hline Death (\%) & 33.7 & 31.1 & 29.6 & 36.4 & 31.3 \\
\hline OR for death & $1.7(1.1-2.6)$ & $1.8(1.1-2.7)$ & NS & NS & NS & NS \\
\hline
\end{tabular}

Figure 1: Box plots showing distribution of age in each ethnic group by gender.

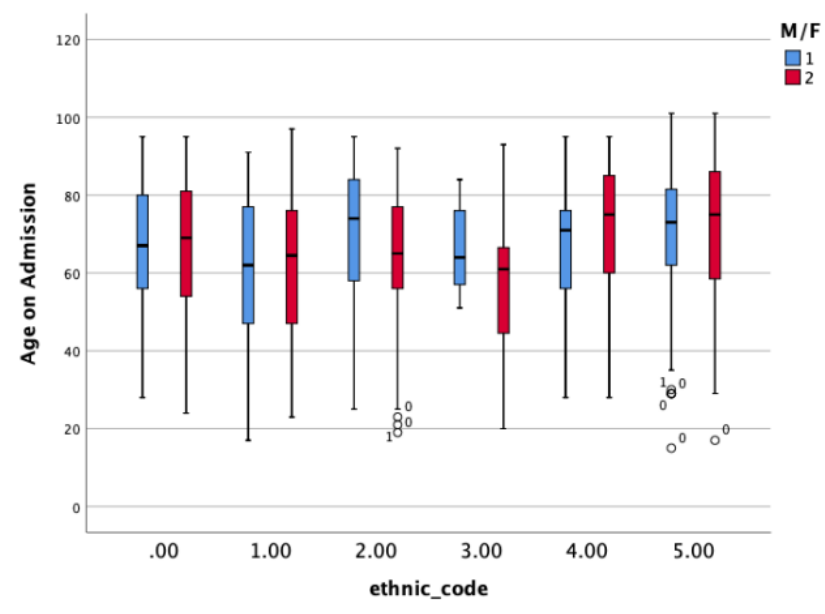

Ethnic groups: $0=$ Not known, $1=$ Asian, $2=$ Black, $3=$ Mixed, $4=$ Other and $5=$ White

Figure 2: Bar chart depicting the proportions of patients with comorbidities in each age-group

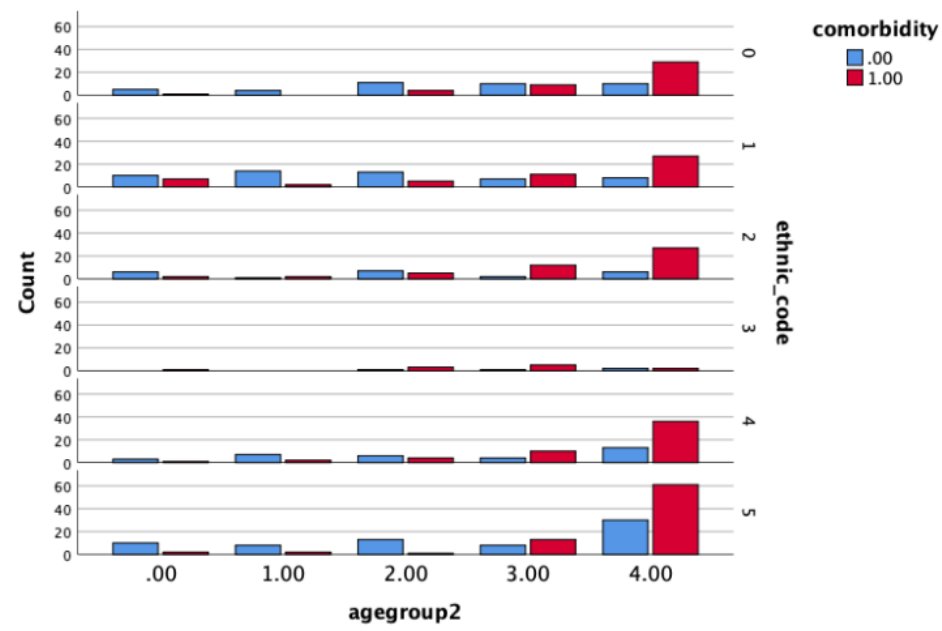

Ethnic groups: $0=$ Not known, $1=$ Asian, $2=$ Black, $3=$ Mixed, $4=$ Other and $5=$ White Age groups; $0=<40,1=40-49,2=50-59,3=60-69$ and $4=>70$ years

Figure 3 indicates the proportion of patients who died or were discharged in each ethnic group based on their age-group. 


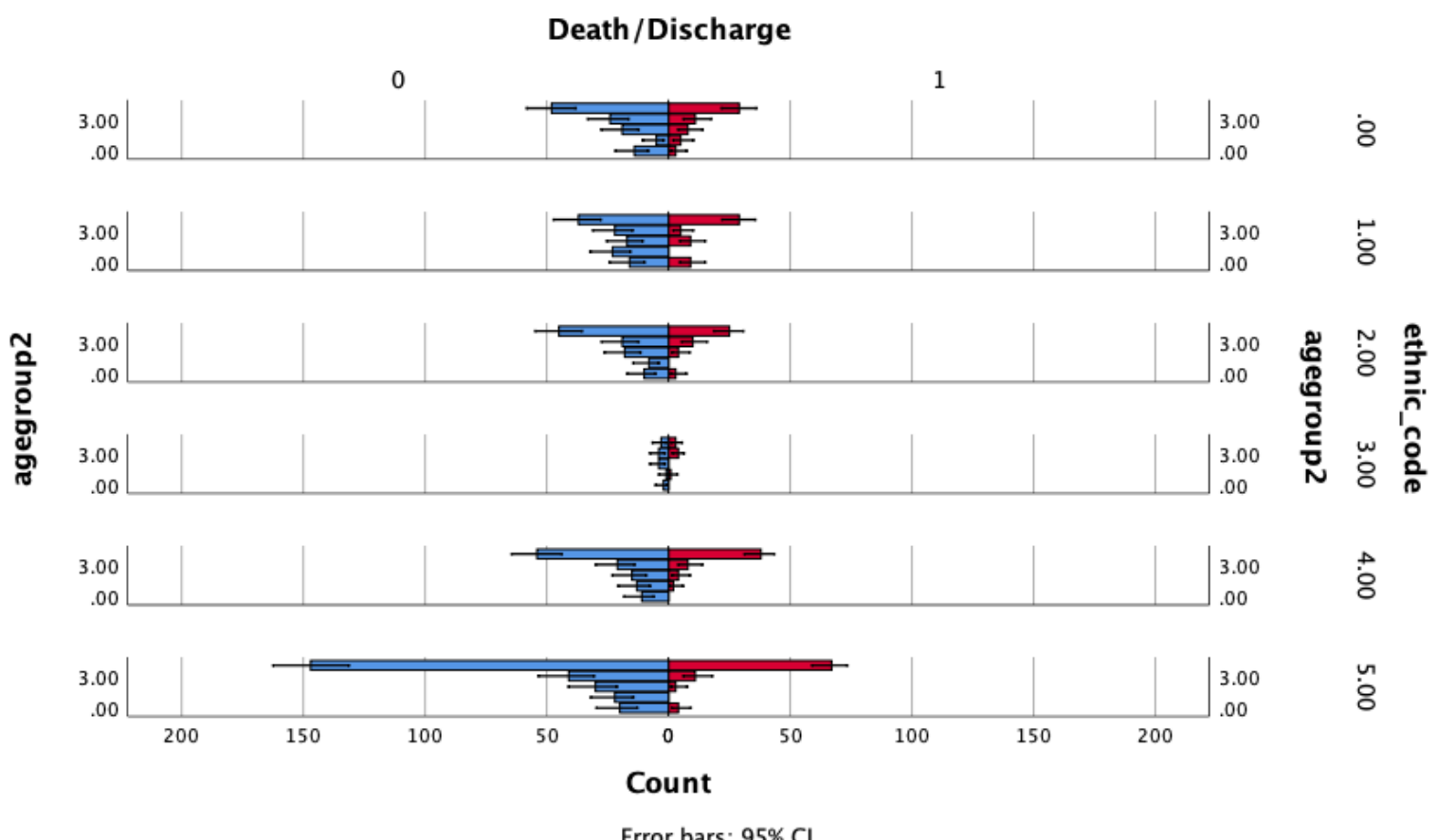

Ethnic groups: $0=$ Not known, $1=$ Asian, $2=$ Black, $3=$ Mixed, $4=$ Other and $5=$ White

Age groups; $0=<40,1=40-49,2=50-59,3=60-69$ and $4=>70$ years

\section{Discussion}

Analysis of data from the UK has demonstrated that ethnicity is an independent risk factor for poor outcomes in patients with COVID-19 (1). The causes are likely to be multi-factorial. London has a higher proportion of ethnic diversity in the local population, thus we conducted this analysis to explore the proportions of each ethnic group represented among the patients admitted to hospital during the COVID-19 surge in the UK.

Our results show that Black and Asian ethnic groups admitted to the hospital with COVID-19 were represented proportionally in relation to local population. In contrast, white patients were under-represented indicating that out of the local population groups, fewer patients from white backgrounds had a diagnosis of COVID-19. Our catchment area has a widely variable population demographic, age distribution, ethnic diversity and social deprivation. Representation of Black ethnic groups vary from $25.9 \%$ in Lambeth to $4.9 \%$ in Sutton, while the Asian ethnic groups make up $6.9 \%$ of the Lambeth population and $16.3 \%$ in Kingston. Hence, for the comparison we have used the Greater London population estimates. (6) However, these results mirror those reported by the analysis of UK NHS digital and ITU data. Patients from BAME backgrounds appear to have a higher rate of hospital admissions, severe disease and death.
Our logistic regression indicates that male sex, age and Asian ethnicity is associated with a higher risk of death from COVID-19. The other key finding is the age distribution, where Asian men and Black women were significantly younger than other groups.

This analysis is limited to patients admitted to hospital, rather than in the whole population. The majority of the COVID-19 cases were community based and did not require testing or hospital stay between March-June, and therefore our report includes patients with higher disease burden. The group of patients classified as 'other-not known' is also a sizeable cohort and seem to sit in the middle of the risk profile. Further exploration of this group and reclassification would help to strengthen our conclusions.

This analysis does not explore the potential causes of the deaths seen in this cohort of patients. UK and international data shows that genetic predisposition, cardiovascular comorbidities as well as socio-economic and racial disparities are likely to play a role (7). The COVID-19 pandemic certainly has highlighted the need for increased awareness of the impact of ethnicity on disease, and has highlighted that there remains significant inequalities within the NHS, an organisation where care is aspired to be universal. It is the responsibility of all individuals and 
organisations within and outside of the NHS to be cognisant of this disparity, and to monitor and adopt measures that will help to balance this injustice.

\section{References}

1. Collaborative TO, Williamson E, Walker AJ, Bhaskaran KJ, Bacon S, Bates C, et al. OpenSAFELY: factors associated with COVID-19-related hospital death in the linked electronic health records of 17 million adult NHS patients. medRxiv. 2020 May 7;2020.05.06.20092999. 2. Pan D, Sze S, Minhas JS, Bangash MN, Pareek N, Divall $P$, et al. The impact of ethnicity on clinical outcomes in COVID-19: A systematic review. EClinicalMedicine [Internet]. 2020 Jun 1 [cited 2020 Aug 16];23. Available from:

https://www.thelancet.com/journals/eclinm/article/PIIS2 589-5370(20)30148-6/abstract

3. Ethnicity and outcomes of coronavirus (COVID19) patients in England [Internet]. NHS Digital. [cited 2020 Aug 14]. Available from: https://digital.nhs.uk/coronavirus/coronavirus-dataservices-updates/ethnicity-and-outcomes-of-covid-19patients-in-england

4. Pareek M, Bangash MN, Pareek N, Pan D, Sze S, Minhas JS, et al. Ethnicity and COVID-19: an urgent public health research priority. The Lancet. 2020 May 2;395(10234):1421-2.

5. Regional ethnic diversity [Internet]. [cited 2020 Aug 14]. Available from: https://www.ethnicity-factsfigures.service.gov.uk/uk-population-byethnicity/national-and-regional-populations/regionalethnic-diversity/latest\#ethnic-groups-by-area 6. London Population 2020 (Demographics, Maps, Graphs) [Internet]. [cited 2020 Aug 15]. Available from: https://worldpopulationreview.com/world-cities/londonpopulation

7. Khunti K, Singh AK, Pareek M, Hanif W. Is ethnicity linked to incidence or outcomes of covid-19? BMJ [Internet]. 2020 Apr 20 [cited 2020 Aug 14];369. Available from:

https://www.bmj.com/content/369/bmj.m1548 